\title{
Effectiveness of Antibiotics: Anti-Bacterial Activity or Microbial Drug Resistance?
}

\author{
Rashed Noor ${ }^{1 *}$, Syeda Muntaka Maniha ${ }^{1}$, Taskina Murshed ${ }^{1}$, M Majibur Rahman ${ }^{2}$ \\ ${ }^{1}$ School of Life Sciences (SLS), Independent University, Bangladesh (IUB), Plot 16, Block B, Aftabuddin Ahmed Road, Bashundhara, Dhaka 1229, Bangladesh, \\ ${ }^{2}$ Department of Microbiology, University of Dhaka, Dhaka 1000, Bangladesh.
}

\begin{abstract}
Antibiotics, both broad- and narrow spectrum, are widely used for treatment of specific infection by a consortium of microorganisms or by a single pathogen, respectively. Oral or intravenous, or even topical administration of different categories of antibiotics in various forms is a common practice round the globe. Yet for the recent years a major public health issue has been raised by the emergence of the drug-resistance microorganisms. A number of researches focused on the issue of the ineffectiveness of antibiotics as well as regarding the evolution of the drugresistance genes within the pathogenic microorganisms. Isolation of the drug-resistant microorganisms including the multi-drug resistant (MDR) and the extensively drug resistant (XDR) bacteria from a range of patients with microbiological infections has been seriously challenging the disease mitigation approaches. Besides, the dominance of the methicillin resistant Staphylococcus aureus (MRSA), vancomycin resistant Staphylococcus aureus (VRSA), etc. are quite frequent as evident from different case studies. Current review focused on the origin and evolution of such drug-resistance incidences, and the promising remedies over the problems of drug-resistance.
\end{abstract}

Keywords: Antibiotics, Drug-resistance, Public health

\section{Introduction}

Antibiotic, for example, penicillin, cephalosporin, streptomycin, tetracycline, gentamicin, griseofulvin, etc. is well defined by Huggo and Russell as the substance produced by a microorganism (mostly Streptomyces species, Penicillium spp., Bacillus, Micromonospora purpurea; Pseudomonas acidophila or Gluconobacter, etc.), or as an analogous material resulting from chemical synthesis (like chloramphenicol, sulphonamides, diaminopyrimidine derivatives, trimoxazole, ntrofuran compounds, quinolones, imidazole derivatives, etc.), which can inhibit the growth of other microorganisms in low concentrations ${ }^{1}$. Eventually, to combat the bacterial and the fungal infection, a range of different categories of antibiotics (including the antibacterial antibiotics like the b-lactam antibiotics, tetracycline group, rifamycins, aminoglycoside-aminocyclitol antibiotics, macrolides, polypeptide antibiotics, glycopeptide antibiotics like vancomycin; or the anti-fungal antibiotics like griseofulvin, polyenes, etc.) has long been practiced as per the physicians' prescriptions marinating the defined forms of administration and dosage $^{1}$. While the liquid forms of antibiotics (i.e., the antibiotic solutions or suspensions) are usually suggested for the pediatric usage, the granular antibiotics are widely taken by the adults following the appropriate drug concentrations as well as the correct dose of administration to get rid of the microbial infection $^{2}$. The industrial manufacturing of antibiotics is of significant importance in order to maintain the quality of the active ingredients of the antibiotics and additionally, it's really important to set the excipients at appropriate concentrations as per the recommendations and guidelines set by the British Pharmacopoeia (BP) or the European Pharmacopoeia (EP), or the United States Pharmacopoeia (USP) ${ }^{3}$. Moreover, as per the guidelines set by the Food and Drug Administration (FDA) as well as by the standard operating procedures (SOPs) set by the pharmaceutical manufacturing and packaging regulatory guidelines (usually guided both by the FDA and the Pharmacopoeia), the total quality management (TQM) is emphasized through the implementation of the good manufacturing practice (GMP) starting from the raw materials ending up to the finished products ready for distribution into the market for sales ${ }^{3-5}$.

The mechanism of different groups of antibiotics against bacteria principally relies on different components of the bacterial cells as the set target sties of specific antibiotic ${ }^{1,6}$. For example, the cell wall of bacteria is commonly known to be prone to attack by the b-lactams, glycopeptides, and cycloserines (isoniazid, ethambutol, etc.). The cell membrane is usually attacked by the polymyxins like polyenes, imidazoles, etc. Ribosome serves as the target site for aminoglycosides, tetracyclines, chloramphenicol, macrolides, fusidic acid, etc. The bacterial chromosome acts as the target site for fluoroquinolones, rifampicin, nitrofurans, etc ${ }^{1}$. However, the onset of microbial drug resistance (MDR) is has been a burning clinical issue since long especially revealed through the multi-drug resistance (MDR) or the extensively drug-resistance (XDR) phenomena whereby a

*Corresponding author:

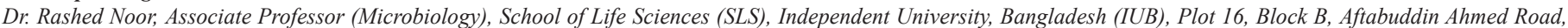
Bashundhara, Dhaka 1229, Bangladesh. Cell: +8801749401451, E-mail: rashednoor@iub.edu.bd 
single microorganism becomes resistant against several types of antibiotics $^{6-12}$. The frequency of the methicillin-resistant $S$. aureus (MRSA), vancomycin-resistant S. aureus (VRSA), vancomycinresistant Enterococcus (VRE) species, penicillin-resistant Streptococcus pneumonia, glycopeptide intermediate sensitivity S. aureus (GISA), the extended-spectrum ${ }^{2}$-lactamase (ESBL) producing bacteria is also increasing ${ }^{1,7-13}$.

Therefore, a number of antibiotics are now appearing ineffective against various pathogenic microorganisms which in turn are increasing the fatality and morbidity around the world especially within the undeveloped or developing countries where the appropriate legislations about the usage of antibiotics are unfortunately lacking ${ }^{14}$. Furthermore, the increasing tendency of the antibiotic resistance certainly sheds light on specific resistance determinants as well as on the drug-resistant microorganisms which are concomitantly spreading both locally and globally, possibly due to the pervasive use of the same antibiotics for human, animal, and in the agricultural sectors ${ }^{15}$. Along these lines, the present review discussed the aspects of the microbial drug-resistance phenomenon against the antibiotics including the causes and effects, and also suggested some possible steps towards minimizing such resistance.

\section{Antibiotic Resistance: Origin and Evolution}

Initially, around 40 years ago, the origins of antibiotic resistance genes were blurred ${ }^{1}$. However, the basic mechanisms of resistance against antibiotics was characterized by the inactivation of the drug, alteration of the target, reduced cellular uptake, and the increased efflux ${ }^{1}$. Afterwards the reasoning behind such resistance were unraveled through the findings of the horizontal acquirement of genes imparting drug-resistance into the bacterial cells; or by the mobilization via insertion sequences, transposons and the conjugative plasmids, by the homologous recombination of the foreign DNA into the chromosomal DNA; or by mutations in the different loci of chromosome $e^{1,16-17}$. It is to be pondered that the antibiotic itself may play a role as the selective marker and secondly the resistance gene acts as the vehicle of resistance; and the resultant impact over time and antibiotic concentration is noticed as the resistance of microorganisms against different antibiotics ${ }^{15}$. In a nearly similar way to Hugo and Russell's work ${ }^{1}$, recently Munita and Arias categorically reported the antibiotic resistance mechanisms again focusing on the modifications of the antibiotic, making the antibiotic excluded by diminishing penetration into the microbial cells, altering the target sites, and certainly by acquiring the adaptation and survival capacity in presence of antibiotic ${ }^{17}$.

Resistance to ${ }^{2}$-lactam antibiotics primarily accounts for the presence of ${ }^{2}$-lactamase, mutation in the penicillin binding protein (PBS); mutation of the ${ }^{2}$-lactam targets and due to the overexpression of efflux pumps ${ }^{1,18}$. Although known, yet it's to ponder that resistance was also noticed to be imparted by plasmids encoding the extended-spectrum b-lactamases (ESBLs) ${ }^{1}$.
Moreover, it should recalled while studying the evolution of the drug-resistance phenomenon that at the early 1950 s, the possession of plasmid encoded b-lactamases had stopped the antibacterial activity of penicillin for the treatment of Staphylococcus aureus infections which led the then scientists to discover the blactamase-stable methicillin (in 1959) ${ }^{1}$. Ironically within a year, the methicillin-resistant $S$. aureus (MRSA) strains evolved; and then vancomycin (the glycopeptide antibiotic) was launched ${ }^{1}$. With a great surprise the resistance even to this antibiotic was noted in 1988 through the identification of the vancomycinresistant enterococci (VRE) ${ }^{1}$.

Microbial resistance against the aminoglycoside antibiotics (streptomycin, kanamycin, gentamicin, tobramycin, apramycin and amikacin) has been reported to be imparted by the enzymatic modification; through increased efflux; and by modifications of the $30 \mathrm{~S}$ ribosomal subunit ${ }^{1,19}$. Another aspect of antibiotic resistance is seen through the colistin-resistant and carbapenemases-producing Klebsiella pneumoniae ${ }^{20-21}$. Indeed the carbapenemases-producing K. pneumoniae became rebellious candidate for the antimicrobial treatment of the hospitalized patients $^{21}$. Resistance against chlortetracycline and oxytetracycline is relatively modern ${ }^{1}$. Shigella flexneri, ESBLproducing Escherichia coli (MRSA), Streptococcus pneumoniae, Klebsiella species and Salmonella enterica serovar typhimurium isolates have been commonly noticed to be resistant against tetracycline ${ }^{1,22}$.

Tetracycline resistance mechanisms have been unraveled through the efflux mechanism, protection of bacterial ribosomes, and finally the inactivation of the antibiotic by defined enzymes ${ }^{22}$. The MDR M. tuberculosis and the efflux-mediated resistance has been identified in case of the fluoroquinolone antibiotics ${ }^{1}$. Resistance to macrolide, lincosamide and streptogramin (MLS) antibiotics have been studied for long while ${ }^{1}$. Fluoroquinolone resistance has been reported to be conferred through a single point mutation wihin the gyrA gene whose product is known to be involved in the DNA supercoiling ${ }^{23}$. This type of resistance is environmentally significant since the accumulation of fluoroquinolone resistance has been evidently shown the formation of biofilm as well as to confer pathogenicity to Campylobacter jejuni ${ }^{23}$. Mechanisms of chloramphenicol resistance has long been known to be mediated by the outer membrane permeability and active efflux in Gram-negartive bacteria; and the resistance is principally conferred by the enzyme acetyltransferase in case of the Gram positive bacterium Staphylococcus epidermidis ${ }^{1,24}$.

Resistance against trimethoprim (TMP) can be caused by the cell wall impermeability (in Klebsiella pneumoniae and Serratia marcescens), alternative metabolic pathways, overproduction of host dihydrofolate reductase (DHFR) and by the mutation in the structural gene for DHFR or by the acquisition of the $d f r$ gene encoding a resistant form (i.e., the alternative DHFR of type I, II or V) as seen in the Enterobacteriaceae ${ }^{1,25}$. Polymyxin B and 
colistin are now a days regarded as the last-resort treatment option because of the abnormal elevation of the MDR Gram-negative bacteria and the carbapenem resistant Pseudomonas aeruginosa, Acinetobacter baumannii, Klebsiella pneumoniae, and Escherichia coli ${ }^{26}$. However, there are reports regarding the dominance of the naturally polymyxin-resistant bacteria including Proteus, Providencia, Morganella, and Serratia ${ }^{26}$. Earlier it was noted the addition of a 4-amino-4-deoxy-L-arabinose (L-Ara4N) moiety to the phosphate groups on the lipid A component of Gramnegative lipopolysaccharide (LPS) conferred the microbial resistance against polymyxin ${ }^{1}$. Resistance against the common drugs; i.e., rifampicin (RIF) and isoniazid (INH), used for the treatment of tuberculosis and the dominance of both MDR- and XDR-tuberculin bacilli have been demonstrated through various studies $^{7-9}$.

\section{Possible Remedies for Minimization of Antibiotic Resistance}

Microbial drug resistance is certainly one of the most dreadful public health issue for the current time when lots of emerging diseases are being disseminated throughout the world ${ }^{14-15,17-19}$. In the environment there may be an interesting framework consisting of both antibiotic-susceptible and the antibioticresistant bacteria which in turn may raise a competition; among which the growth of the antibiotic-resistant strains may be enhanced through the excessive usage of antibiotics ${ }^{15}$. In order to restore the efficacy of the antibiotics to their earlier forms as well as to achieve the efficacy of the new antibiotic molecules, a precise rationale of antibiotic usage has to be kept which in turn would esteem the antibiotic sensitive bacteria ${ }^{15}$. Besides treatment/ chemotherapeutic purpose, the unreasonable use of antibiotics in agriculture should be minimized since the over dosage of antibiotics have been correlated with the dominance of an array of pathogenic drug-resistant microorganisms ${ }^{18}$. A recent interesting aspect on controlling the antibiotic exposure with a motive to minimize the drug-resistance phenomenon has been seen through the suggestion of generating the evidence based policies by using mathematical models which can play as the key drivers of the drug-resistance transmission dynamics in a complicated infection ${ }^{27}$. Such modelling also focuses on the evolutionary processes of the microbial drug resistance. Of course the challenges associated with measuring the antibiotic resistance evolution using such mathematical models needs to be further chalked out, together with translating the mathematical modelling evidence into policy ${ }^{27}$.

Laboratory research based on the random examination of antibacterial activities of different antibiotics can be exercised as a collaborative means with either the industrial manufacturing and packaging pharmaceutical companies or the diagnostic centers ${ }^{6}$. Community base remedies about the misuse of antibiotics can be implemented like putting the restrictions in the selling of antibiotics without physicians' prescriptions. Antibiotics should not be advised by any physician at the very beginning of any suspected infection; rather the diagnosis needs to be conducted first. No antibiotics should be suggested for simple cold, cough, sneezing, etc. Without the evidence of bacterial infection, no antibiotics should be routinely prescribed for sore throat or the acute otitis media and sinusitis. Besides, in hospitals, physicians need to be very careful about the routine use of antibiotics for surgical prophylaxis which should be actually minimal ${ }^{28}$.

Indeed the presence of an antibiotic is thought to be a selection marker for a high frequency of microorganisms resistant to that specific antibiotic since resistance to any antibiotic treatment actually imparts the microbial strains a benefit compared to the antibiotic susceptible strains ${ }^{27,29}$. This leads to a bit strange situation that the drug-resistant bacteria may possess lower survival capacity within the host in absence of antibiotics ${ }^{27,30}$. Lack of understanding of such selection pressure for the antibiotic resistance is a major challenge to resolve the problem of the microbial drug resistance. Physicians should rethink on such facts to actually find out the root causes for the antibiotic resistance; and should focus more on the drug-resistance resulting from the case studies and diagnostic tests.

\section{Conclusion}

Microbial drug-resistance is a major public health throughout the world; and it's one of the greatest threats to the success of the modern Medical Science. The dominance of the MDR or XDR bacteria within the environment as well as the transmission of the drug resistance genes in certain ecological niches is gradually increasing. In addition, the intense genetic agility of microorganisms especially of the pathogenic ones that activate defined responses in course of mutational tolerances, or the amendment of gene expression imparting the resistance to multiple antibiotics. Eventually, such a situation brings failure in the chemotherapy or in the treatment of any specific disease. Therefore, perceptive knowledge on the genetic makeup of the associated factors is of significance to devise strategies to diminish or decrease the emergence and subsequent stretching of such drug-resistance.

\section{References}

1. Denyer S, Hodges NA and Gorman SP. 2004. Hugo and Russell's Pharmaceutical Microbiology, $7^{\text {th }}$ edn. Blackwell Scientific Publications, Oxford.

2. Al-Ramahi RJ, Zaid AA and Anabousi H. 2015. Problems associated with reconstitution, administration, and storage of antibiotic suspensions for pediatrics: A cross-sectional study in Nablus city, Palestine. BMC Res Notes. 8: 760 .

3. Noor R, Zerin N and Das KK. 2015. Microbiological quality of pharmaceutical products in Bangladesh: Current research perspective. Asian Pac J Trop Dis. 5(4): 264-270.

4. Cundell AM. 2005. Managing the microbiological quality of pharmaceutical excipients. PDA J Pharma Sci Technol. 59(6): 381-395.

5. Underwood E. 1998. Good manufacturing practice. In Principles and Practice of Disinfection, Preservation and Sterilization (Russell AD, Hugo WB and Ayliffe GAJ eds.). Blackwell Scientific, Oxford.

6. Dutta S, Hasan MR, Rahman F, Jilani MSA and Noor R. 2013. Study of antimicrobial susceptibility of clinically significant microorganisms 
isolated from selected areas of Dhaka, Bangladesh. Bangladesh J Med Sci. 12(1): 34-42.

7. Noor R, Hossain A, Munshi SK, Rahman F and Kamal SM. 2013. Slide drug susceptibility test for the detection of multidrug-resistant tuberculosis in Bangladesh. J Infect Chemother. 19(5): 818-824.

8. Noor R, Akhter S, Rahman F, Munshi SK, Kamal SMM and Feroz F. 2013. Frequency of extensively drug-resistant tuberculosis (XDR-TB) among re-treatment cases in NIDCH, Dhaka, Bangladesh. $J$ Infect Chemother. 19(2): 243-248.

9. Aurin TH, Munshi SK, Kamal SM, Rahman MM, Hossain MS, Marma T, Rahman F and Noor R. 2014. Molecular approaches for detection of the multi-drug resistant tuberculosis (MDR-TB) in Bangladesh. PLOS ONE. 9(6): e99810.

10. Hasan R, Acharjee M and Noor R. 2016. Prevalence of Vancomycin resistant Staphylococcus aureus (VRSA) in methicillin resistant S. aureus (MRSA) strains isolated from burn wound infections. Tzu Chi Med J. 28(2): 49-53.

11. Khan SA, Feroz F and Noor R. 2013. Study of extended spectrum ${ }^{2-}$ lactamase producing bacteria from urinary tract infection in Dhaka city, Bangladesh. Tzu Chi Med J. 25(1): 39-42.

12. Zarifan AR, Askari E, Pourmand MR and Nasab MN. 2012. High-level vancomycin-resistant Staphylococcus aureus (VRSA) in Iran: a systematic review. J Med Bacteriol. 1(3-4): 53-61.

13. Ranjbar R and Farahani A. 2019. Study of genetic diversity, biofilm formation, and detection of carbapenemase, MBL, ESBL, and tetracycline resistance genes in multidrug-resistant Acinetobacter baumannii isolated from burn wound infections in Iran. Antimicrob Resist Infect Control. 8: 172.

14. Noor R and Munna MS. 2015. Emerging diseases in Bangladesh: Current microbiological research perspective. Tzu Chi Med J. 27(2): 49-53.

15. Levy SB. 2007. Antibiotic Resistance: An Ecological Imbalance. Novartis Foundation Symposia, 1-14.

16. Chowdhury FFK, Acharjee M and Noor R. 2015. Maintenance of environmental sustainability through microbiological study of pharmaceutical solid wastes. Clean-Soil Air Water. 44(3): 309-316.

17. Munita JM and Arias CA. 2016. Mechanisms of antibiotic resistance. Microbiol Spectrum. 4(2): 10.1128/microbiolspec.VMBF-0016-2015.

18. Lingzhi L, Haojie G, Dan G, Hongmei M, Yang L, Mengdie J, Chengkun $\mathrm{Z}$ and Xiaohui Z. 2018. The role of two-component regulatory system in 2-lactam antibiotics resistance. Microbiol Res. 215: 126-129.
19. Doi Y, Wachino JI and Arakawa Y. 2016. Aminoglycoside Resistance: The Emergence of Acquired 16S Ribosomal RNA Methyltransferases. Infectious disease clinics of North America. 30(2): 523-537.

20. Hayder N, Hasan Z, Afrin S and Noor R. 2013. Determination of the frequency of carbapenemase producing Klebsiella pneumoniae isolates in Dhaka city, Bangladesh. Stamford J Microbiol. 2(1): 28-30.

21. di Tella D, Tamburro M, Guerrizio G, Fanelli I, Sammarco ML and Ripabelli G. 2019. Molecular Epidemiological Insights into ColistinResistant and Carbapenemases-Producing Clinical Klebsiella pneumoniae Isolates. Infect Drug Resist. 12: 3783-3795.

22. Grossman TH. 2016. Tetracycline antibiotics and resistance. Cold Spring Harbor Perspectives in Medicine. 6(4): a025387.

23. Whelan M, Ardill L, Koide K, Nakajima C, Suzuki Y, Simpson JC and Ó Cróinín T. 2019. Acquisition of fluoroquinolone resistance leads to increased biofilm formation and pathogenicity in Campylobacter jejuni. Scientific Reports. 9(1): 18216.

24. Shaw WV, Bentley DW and Sands L. 1970. Mechanism of chloramphenicol resistance in Staphylococcus epidermidis. J Bacteriol. 104(3): 1095-1105.

25. Huovinen P. 1987. Trimethoprim resistance. Antimicrob Agents Chemother. 31(10): 1451-1456.

26. Olaitan AO, Morand S and Rolain JM. 2014. Mechanisms of polymyxin resistance: acquired and intrinsic resistance in bacteria. Frontiers Microbiol. 5: 643.

27. Knight GM, Davies NG, Colijn C, Coll F, Donker T, Gifford DR, Glover RE, Jit M, Klemm E, Lehtinen S, Lindsay JA, Lipsitch M, Llewelyn MJ, Mateus ALP, Robotham JV, Sharland M, Stekel D, Yakob L and Atkins KE. 2019. Mathematical modelling for antibiotic resistance control policy: do we know enough? BMC Infect Dis. 19: 1011.

28. Standing Medical Advisory Committee, Department of Health. The path of least resistance. London, HMSO, 1998.

29. Davies J and Davies D. 2010. Origins and evolution of antibiotic resistance. Microbiol Mol Biol Rev. 74(3): 417-33.

30. Niewiadomska AM, Jayabalasingham B, Seidman JC, Willem L, Grenfell B, Spiro D and Viboud C. 2019. Population-level mathematical modeling of antimicrobial resistance: a systematic review. BMC Med. 17(1): 81 . 